\title{
Discovery of VHE $\gamma$-rays from the blazar 1ES 1215+303 with the MAGIC telescopes and simultaneous multi-wavelength observations
}
J. Aleksić ${ }^{1}$, E. A. Alvarez ${ }^{2}$, L. A. Antonelli ${ }^{3}$, P. Antoranz ${ }^{4}$, S. Ansoldi ${ }^{15}$, M. Asensio ${ }^{2}$, M. Backes ${ }^{5}$ U. Barres de Almeida ${ }^{6}$, J. A. Barrio ${ }^{2}$, D. Bastieri ${ }^{7}$, J. Becerra González ${ }^{8,31, *}$, W. Bednarek ${ }^{9}$, K. Berger ${ }^{8,10}$, E. Bernardini ${ }^{11}$, A. Biland ${ }^{12}$, O. Blanch ${ }^{1}$, R. K. Bock ${ }^{6}$, A. Boller ${ }^{12}$, G. Bonnoli ${ }^{3}$, D. Borla Tridon ${ }^{6}$, T. Bretz ${ }^{13,27}$, A. Cañellas ${ }^{14}$, E. Carmona ${ }^{6,29}$, A. Carosi ${ }^{3}$, P. Colin ${ }^{6, *}$, E. Colombo ${ }^{8}$, J. L. Contreras ${ }^{2}$, J. Cortina ${ }^{1}$, L. Cossio ${ }^{15}$, S. Covino ${ }^{3}$, P. Da Vela ${ }^{4}$, F. Dazzi ${ }^{15,28}$, A. De Angelis ${ }^{15}$, G. De Caneva ${ }^{11}$, E. De Cea del Pozo ${ }^{16}$, B. De Lotto ${ }^{15}$, C. Delgado Mendez ${ }^{8,29}$, A. Diago Ortega ${ }^{8,10}$, M. Doert ${ }^{5}$, A. Domínguez ${ }^{17}$, D. Dominis Prester ${ }^{18}$, D. Dorner $^{12}$, M. Doro ${ }^{19}$, D. Eisenacher ${ }^{13}$, D. Elsaesser ${ }^{13}$, D. Ferenc ${ }^{18}$, M. V. Fonseca ${ }^{2}$, L. Font ${ }^{19}$, C. Fruck ${ }^{6}$, R. J. García López ${ }^{8,10}$, M. Garczarczyk ${ }^{8}$, D. Garrido Terrats ${ }^{19}$, M. Gaug $^{19}$, G. Giavitto ${ }^{1}$, N. Godinović ${ }^{18}$, A. González Muñoz ${ }^{1}$, S. R. Gozzini ${ }^{11}$, D. Hadasch ${ }^{16}$, D. Häfner ${ }^{6}$, A. Herrero ${ }^{8,10}$, D. Hildebrand ${ }^{12}$, J. Hose ${ }^{6}$, D. Hrupec ${ }^{18}$, B. Huber ${ }^{12}$, F. Jankowski ${ }^{11}$, T. Jogler ${ }^{6,30}$, V. Kadenius ${ }^{20}$, H. Kellermann ${ }^{6}$, S. Klepser ${ }^{1}$, T. Krähenbühl ${ }^{12}$, J. Krause ${ }^{6}$, A. La Barbera ${ }^{3}$, D. Lelas ${ }^{18}$, E. Leonardo ${ }^{4}$, N. Lewandowska ${ }^{13}$, E. Lindfors ${ }^{20}{ }^{*}$, S. Lombardi $^{7}{ }^{*}$, M. López ${ }^{2}$, R. López-Coto $^{1}$, A. López-Oramas ${ }^{1}$, E. Lorenz ${ }^{6,12}$, M. Makariev ${ }^{21}$, G. Maneva ${ }^{21}$, N. Mankuzhiyil ${ }^{15}$, K. Mannheim ${ }^{13}$, L. Maraschi ${ }^{3}$, M. Mariotti ${ }^{7}$, M. Martínez ${ }^{1}$, D. Mazin ${ }^{1}, 6$, M. Meucci $^{4}$, J. M. Miranda ${ }^{4}$, R. Mirzoyan ${ }^{6}$, J. Moldón ${ }^{14}$, A. Moralejo ${ }^{1}$, P. Munar-Adrover ${ }^{14}$, A. Niedzwiecki ${ }^{9}$, D. Nieto ${ }^{2}$, K. Nilsson ${ }^{20,32}$, N. Nowak ${ }^{6}$, R. Orito ${ }^{22}$, S. Paiano ${ }^{7}$, D. Paneque ${ }^{6}$, R. Paoletti ${ }^{4}$, S. Pardo ${ }^{2}$, J. M. Paredes ${ }^{14}$, S. Partini ${ }^{4}$, M. A. Perez-Torres ${ }^{1}$, M. Persic ${ }^{15,23}$, M. Pilia ${ }^{24}$, J. Pochon $^{8}$, F. Prada ${ }^{17}$, P. G. Prada Moroni ${ }^{25}$, E. Prandini ${ }^{7}$, I. Puerto Gimenez ${ }^{8}$, I. Puljak ${ }^{18}$, I. Reichardt ${ }^{1}$, R. Reinthal ${ }^{20}$, W. Rhode $^{5}$, M. Ribó ${ }^{14}$, J. Rico ${ }^{26,1}$, S. Rügamer ${ }^{13}$, A. Saggion ${ }^{7}$, K. Saito ${ }^{6}$, T. Y. Saito ${ }^{6}$, M. Salvati ${ }^{3}$, K. Satalecka ${ }^{2}$, V. Scalzotto ${ }^{7}$, V. Scapin ${ }^{2}$, C. Schultz ${ }^{7}$, T. Schweizer ${ }^{6}$, S. N. Shore ${ }^{25}$, A. Sillanpää ${ }^{20}$, J. Sitarek ${ }^{1,9, *}$, I. Snidaric ${ }^{18}$, D. Sobczynska ${ }^{9}$,

F. Spanier ${ }^{13}$, S. Spiro ${ }^{3}$, V. Stamatescu ${ }^{1}$, A. Stamerra ${ }^{4}$, B. Steinke 6 , J. Storz ${ }^{13}$, N. Strah ${ }^{5}$, S. Sun ${ }^{6}$, T. Surić ${ }^{18}$,

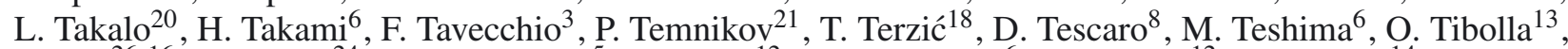
D. F. Torres ${ }^{26,16}$, A. Treves ${ }^{24}$, M. Uellenbeck ${ }^{5}$, P. Vogler ${ }^{12}$, R. M. Wagner ${ }^{6}$, Q. Weitzel ${ }^{12}$, V. Zabalza ${ }^{14}$, F. Zandanel ${ }^{17}$, R. Zanin ${ }^{14}$ (the MAGIC Collaboration), A. Berdyugin ${ }^{20,32}$, S. Buson ${ }^{7}$, E. Järvelä ${ }^{33}$, S. Larsson ${ }^{34,35,36}$, A. Lähteenmäki ${ }^{33}$, and J. Tammi ${ }^{33}$

(Affiliations can be found after the references)

Received 29 February 2012 / Accepted 22 May 2012

\section{ABSTRACT}

Context. We present the discovery of very high energy (VHE, $E>100 \mathrm{GeV}$ ) $\gamma$-ray emission from the BL Lac object $1 \mathrm{ES} 1215+303$ by the MAGIC telescopes and simultaneous multi-wavelength data in a broad energy range from radio to $\gamma$-rays.

Aims. We study the VHE $\gamma$-ray emission from 1ES $1215+303$ and its relation to the emissions in other wavelengths.

Methods. Triggered by an optical outburst, MAGIC observed the source in 2011 January-February for $20.3 \mathrm{~h}$. The target was monitored in the optical $R$-band by the KVA telescope that also performed optical polarization measurements. We triggered target of opportunity observations with the Swift satellite and obtained simultaneous and quasi-simultaneous data from the Fermi Large Area Telescope and from the Metsähovi radio telescope. We also present the analysis of older MAGIC data taken in 2010.

Results. The MAGIC observations of 1ES 1215+303 carried out in 2011 January-February resulted in the first detection of the source at VHE with a statistical significance of $9.4 \sigma$. Simultaneously, the source was observed in a high optical and X-ray state. In 2010 the source was observed in a lower state in optical, X-ray, and VHE, while the GeV $\gamma$-ray flux and the radio flux were comparable in 2010 and 2011 . The spectral energy distribution obtained with the 2011 data can be modeled with a simple one zone SSC model, but it requires extreme values for the Doppler factor or the electron energy distribution.

Key words. BL Lacertae objects: individual: 1ES 1215+303 - gamma-rays: galaxies

\section{Introduction}

Most of the extragalactic sources from which very high energy $(\mathrm{VHE},>100 \mathrm{GeV}) \gamma$-ray emissions has been detected are blazars. These objects are commonly believed to be a subtype of active galactic nuclei (AGN) whose relativistic jet points very close to the line of sight of the observer. Blazars are characterized by high amplitude variability at all wavebands from radio to $\gamma$-rays. The correlations between the different energy bands are complicated, but in general it seems that high states in lower 
energy bands (e.g. optical) are accompanied by high states in the higher energies (i.e. $\gamma$-rays) at least in some sources (see e.g. Reinthal et al. 2012).

The spectral energy distribution (SED) of blazars exhibits a generic two-bump structure: one peak with a maximum in the spectral range from radio to X-rays and a second peak in the interval from X-ray to $\gamma$-ray. The radiation is produced in a highly beamed plasma jet and the double peaked SED is often explained by a single population of relativistic electrons. The first peak is due to synchrotron emission in the magnetic field of the jet and the second peak is caused by inverse Compton (IC) scattering of low-energy photons (Rees 1967). The low-energy photons can originate externally to the jet (external Compton scattering, Dermer \& Schlickeiser 1993) or be produced within the jet via synchrotron radiation (synchrotron self-Compton scattering, SSC, Maraschi et al. 1992).

Blazar is a common term used for flat spectrum radio quasars (FSRQs) and BL Lac objects (BL Lacs), which are thought to be intrinsically different. The FSRQs show broad emission lines in their optical spectra while the BL Lacs have featureless spectra with weak or no emission lines possibly masked by a strong emission from the jet. This indicates that in BL Lac objects the main population of seed photons for Compton scattering should originate from the synchrotron emission. Indeed most of the SEDs of BL Lacs are well described with simple SSC model (e.g. Bloom \& Marscher 1996; Tavecchio et al. 1998).

MAGIC has been successfully performing optically triggered VHE $\gamma$-ray observations of AGN since the start of its operations. The triggers have been provided by the Tuorla blazar monitoring program ${ }^{1}$ and the target of opportunity (ToO) observations with MAGIC have resulted in the discovery of five new VHE $\gamma$-ray emitting sources (Mrk 180, Albert et al. 2006b; 1ES 1011+496, Albert et al. 2007; S5 0716+714, Anderhub et al. 2009; B3 2247+381, Aleksić et al. 2012a; and 1ES $1215+303$, this paper). However, in many cases it has not been possible to confirm with high statistical significance if the sources were in higher VHE $\gamma$-ray state than usual during the observations. The long-term studies of individual VHE $\gamma$-ray blazars like Mrk 421 (Acciari et al. 2011) and PG 1553+113 (Aleksić et al. 2012b) have also yielded controversial results on the correlation between the two broad energy ranges. Thus, to date, the connection between the optical and VHE $\gamma$-ray states has remained an open question.

1ES 1215+303 (also known as ON 325) is a high synchrotron peaking BL Lac object (Abdo et al. 2010b) with redshift $z=0.130$ (Akiyama et al. 2003, however, $z=0.237$ is also reported in the literature, e.g. $\mathrm{NED}^{2}$ ). The source was classified as a promising candidate $\mathrm{TeV}$ blazar (Costamante \& Ghisellini 2002; Tavecchio et al. 2010) and has been observed several times in VHE $\gamma$-rays before the observations presented here. The previous observations yielded only upper limits, Whipple: $F(>430 \mathrm{GeV})<1.89 \times 10^{-11} \mathrm{~cm}^{-2} \mathrm{~s}^{-1}$ (Horan et al. 2004); MAGIC: $F(>120 \mathrm{GeV})<3.5 \times 10^{-11} \mathrm{~cm}^{-2} \mathrm{~s}^{-1}$ (Aleksić et al. 2011a). The source was listed in the Fermi Large Area Telescope (LAT) bright AGN catalog (Abdo et al. 2009) as showing a hard spectrum $(\Gamma=1.89 \pm 0.06)$. It underwent a large outburst in late 2008, and in this catalog 1 ES $1215+303$ is the only high energy peaking source that shows significant variability. In the second Fermi-LAT AGN catalog (Ackermann et al. 2011) other high synchrotron peaking sources have also been flagged as variable.

\footnotetext{
1 http://users.utu.fi/kani/1m/

2 http://nedwww.ipac. caltech.edu/
}

In the first days of 2011 January 1ES 1215+303 was observed to be in a high optical state. This triggered MAGIC observations, extending until 2011 February, that resulted in the discovery of VHE $\gamma$-rays from the source (Mariotti 2011). In this paper we present the results of the 2011 January-February observations. We also present the previous observations of 1ES $1215+303$ with the MAGIC telescopes performed in 2010 January-February and 2010 May-June that produced only a hint of signal. For all epochs we present simultaneous and quasi-simultaneous multi-wavelength data from radio, optical, X-ray, and $\mathrm{GeV} \gamma$-rays.

\section{Observations and data analysis}

The observations of 1ES $1215+303$ were performed in a broad wavelength range (from radio to VHE $\gamma$-rays) by 5 different instruments. This is the first time that such a broad wavelength range is covered for this source in quasi-simultaneous observations.

\subsection{MAGIC}

MAGIC consists of two $17 \mathrm{~m}$ Imaging Air Cherenkov Telescopes (IACTs) sensitive to $\gamma$-rays with energy above $50 \mathrm{GeV}$ in standard trigger mode (which is the lowest trigger energy threshold among the existing IACTs). The system is located in the Canary Island of La Palma, $2200 \mathrm{~m}$ above sea level. Since fall 2009 the telescopes are working together in stereoscopic mode which ensures a sensitivity of $<0.8 \%$ of the Crab Nebula flux above $300 \mathrm{GeV}$ in $50 \mathrm{~h}$ of observations (Aleksić et al. 2012c). The field of view of the each MAGIC camera has a diameter of $3.5^{\circ}$.

1ES $1215+303$ was observed by MAGIC in 2010 JanuaryFebruary, 2010 May-June, and 2011 January-February, for a total of $48 \mathrm{~h}$. The observations were done in the so-called wobble mode (i.e. with the source offset by $0.4^{\circ}$ from the camera center), which provides a simultaneous estimate of the background from the same data set (Fomin et al. 1994). While most of the data were taken in dark night conditions, a small fraction were taken in presence of moderate moonlight. The data span a range of zenith angle from $1^{\circ}$ to $40^{\circ}$ with most of the data taken below $25^{\circ}$ (in 2010 the mean zenith angle was $\sim 13^{\circ}$, and in $2011 \sim 8^{\circ}$ ).

The data were analyzed using the standard MAGIC software and analysis package (Aleksić et al. 2012c). Another VHE $\gamma$-ray emitter, 1ES $1218+304$ (Albert et al. 2006a) is present in the same field of view as 1ES $1215+303$. The sources are separated by $\sim 0.8^{\circ}$, which is much larger than the point spread function (PSF) of the MAGIC telescopes $\left(\sim 0.1^{\circ}\right)$, so there was no source confusion or contamination. However, these sources have nearly the same Right Ascension, so in the standard wobble setup used in the 2010 January-February observations, the background estimation region partially overlapped with the 1ES $1218+304$ position. This would result in an overestimate of the background, so this region was excluded from the background estimate. In the later observations (2010 May-June, and 2011 January-February) the wobbling offset direction was changed to have the standard background estimation regions far from the second source.

After the data quality selection, based mainly on the rate of stereo events, the data samples of January-February 2010, May-June 2010 and January-February 2011 contain 19.4, 3.5, and $20.6 \mathrm{~h}$ of good quality data respectively. Because of the different positions of the source in the camera, and the variable nature of AGN, we decided to split the analysis into these 3 periods. 


\subsection{Fermi-LAT}

The Fermi-LAT is a pair conversion telescope designed to cover the energy band from $20 \mathrm{MeV}$ to greater than $300 \mathrm{GeV}$. It operates in all-sky survey mode and therefore can provide observations of 1ES $1215+303$ simultaneous to MAGIC. In this paper the standard LAT Science Tools (version v9r23p0) were used to analyze the data collected in the time interval from 2008 August 5 to 2011 March 22. For this analysis, only events belonging to the "Diffuse" class (which have the highest probability of being photons) and located in a circular Region Of Interest (ROI) of $7^{\circ}$ radius, centered at the position of $1 \mathrm{ES} 1215+303$, were selected (using Pass 6 event selection). In addition, we applied a cut on the zenith angle $\left(<100^{\circ}\right)$ limb $\gamma$-rays and a cut on the rocking angle $\left(>52^{\circ}\right)$ to limit Earth limb contamination.

The data analysis of $1 \mathrm{ES} 1215+303$ is very challenging due to the presence of several $\gamma$-ray emitting sources in the same ROI. 1ES $1218+304$ is located at a distance of just $0.8^{\circ}$ from the source of interest. Another well known VHE emitter, W Comae, is located at $\sim 2^{\circ}$ from the latter source. Thus, the LAT analysis was restricted to energies above $1 \mathrm{GeV}$ where the Fermi-LAT PSF is sufficiently narrow ${ }^{3}$ to separate 1ES $1215+303$ from the other sources in the ROI. The unbinned likelihood method was applied to events in the energy range from $1 \mathrm{GeV}$ to $300 \mathrm{GeV}$. All point sources from the 2FGL catalog (Nolan et al. 2012) located within $12^{\circ}$ of $1 \mathrm{ES} 1215+303$ were included in the model of the region. Sources located within a $5^{\circ}$ radius centered on 1 ES $1215+303$ position had their flux and photon index left as free parameters. The diffuse Galactic and isotropic components (including residual instrumental background) were modeled with the publicly available files gll_iem_v02_P6_V11_DIFFUSE.fit and isotropic iem_vQ2_P6_V11_DIFFUSE.txt ${ }^{4}$. The normalizations of the components comprising the total background model were allowed to vary freely during the spectral point fitting. The instrument response functions P6_V11_DIFFUSE were used. The successful separation of flux between 1ES 1215+303 and 1ES $1218+304$ was verified by the absence of any significant correlation between their light curves. The systematic uncertainty in the flux is estimated as $5 \%$ at $560 \mathrm{MeV}$ and $20 \%$ at $10 \mathrm{GeV}$ and above ${ }^{5}$.

\subsection{Swift}

The Swift satellite (Gehrels et al. 2004) is equipped with three telescopes, the Burst Alert Telescope (BAT; Barthelmy et al. 2005 ) covering the $15-150 \mathrm{keV}$ energy range, the X-ray telescope (XRT; Burrows et al. 2005) covering the $0.2-10 \mathrm{keV}$ energy band, and the UV/Optical Telescope (UVOT; Roming et al. 2005 ) covering the $180-600 \mathrm{~nm}$ wavelength range.

A Swift ToO request was submitted on 2011 January 3. The Swift observations started on January 4 until January 12 with four $\sim 5 \mathrm{ks}$ exposures in photon counting mode. The data were processed with standard procedures using the FTOOLS task XRTPIPELINE (version 0.12.6) distributed by HEASARC within the HEASoft package (v.6.10). Events with grades 0-12 were selected for the data (see Burrows et al. 2005) and the latest response matrices available in the Swift CALDB (v.20100802)

\footnotetext{
3 http://www.slac.stanford.edu/exp/glast/groups/canda/ archive/pass6v11/lat_Performance.htm

4 http://fermi.gsfc.nasa.gov/ssc/data/access/lat/ BackgroundModels.html

5 http://fermi.gsfc.nasa.gov/ssc/data/analysis/LAT_ caveats.html
}

were used. For the spectral analysis, we extracted the source events in the $0.3-10 \mathrm{keV}$ range within a circle with a radius of 20 pixels ( $\sim 7 \mathrm{arcsec})$. The background was extracted from an off-source circular region with a radius of 40 pixels.

The spectra were extracted from the corresponding event files and binned using GRPPHA to ensure a minimum of 25 counts per energy bin, in order to obtain reliable $\chi^{2}$ statistics. Spectral analysis was performed using XSPEC version 12.6.0. The neutral hydrogen-equivalent column density was fixed to the Galactic value in the direction of the source $1.74 \times 10^{20} \mathrm{~cm}^{-2}$ (Kalberla et al. 2005).

Swift/UVOT observed the source with all filters $(V, B, U$, $U V W 1, U V M 2, U V W 2$ ) for four nights. UVOT source counts were extracted from a circular region 5 arcsec-sized centered on the source position, while the background was extracted from a larger circular nearby source-free region. These data were processed with the uvotmaghist task of the HEASOFT package. The observed magnitudes have been corrected for Galactic extinction $E_{B-V}=0.024 \mathrm{mag}$ (Schlegel et al. 1998), applying the formulae by Pei (1992) and finally converted into fluxes following Poole et al. (2008).

\subsection{KVA}

The KVA optical telescopes are located in La Palma, but are operated remotely from Finland. The two telescopes are attached to the same fork. The larger telescope has a mirror diameter of $60 \mathrm{~cm}$ and the smaller $35 \mathrm{~cm}$.

The $35 \mathrm{~cm}$ telescope is used for simultaneous photometric observations with MAGIC, but also to monitor potential VHE $\gamma$-ray candidate AGN in order to trigger MAGIC observations if the sources are in high optical states. The observations are performed in the $R$-band and the magnitude of the source is measured from CCD images using differential photometry. The comparison star magnitudes for 1ES 1215+303 are from Fiorucci \& Tosti (1996), and the magnitudes are converted to flux using the formula and values from Bessell (1979). 1ES $1215+303$ has been observed regularly as part of the Tuorla blazar monitoring program since 2002.

The $60 \mathrm{~cm}$ telescope is used for polarimetric observations (see e.g. Piirola et al. 2005; Aleksić et al. 2011b). For 1ES $1215+303$ polarimetric observations were performed on six nights from 2011 January 7 to January 17. The degree of polarization and position angle were calculated from the intensity ratios of the ordinary and extraordinary beams using standard formulae and semiautomatic software specially developed for polarization monitoring purposes.

\subsection{Metsähovi radio telescope}

$37 \mathrm{GHz}$ radio observations were made with the $13.7 \mathrm{~m}$ Metsähovi radio telescope located in Kylmälä, Finland. The telescope, the observation methods, and the data analysis procedure are described in e.g. Teräsranta et al. (1998). The telescope detection limit is $\sim 0.2 \mathrm{Jy}$ under optimal conditions and since $1 \mathrm{ES} 1215+303$ is a rather weak source at $37 \mathrm{GHz}$ it can only be observed under good weather conditions. Typically, an acceptable measurement of the source is obtained approximately once per month. Data were obtained simultaneously with the MAGIC observations in 2010 June, but in 2011 January-February the weather did not allow simultaneous observations with MAGIC, the closest points being from 2010 December and 2011 March. 

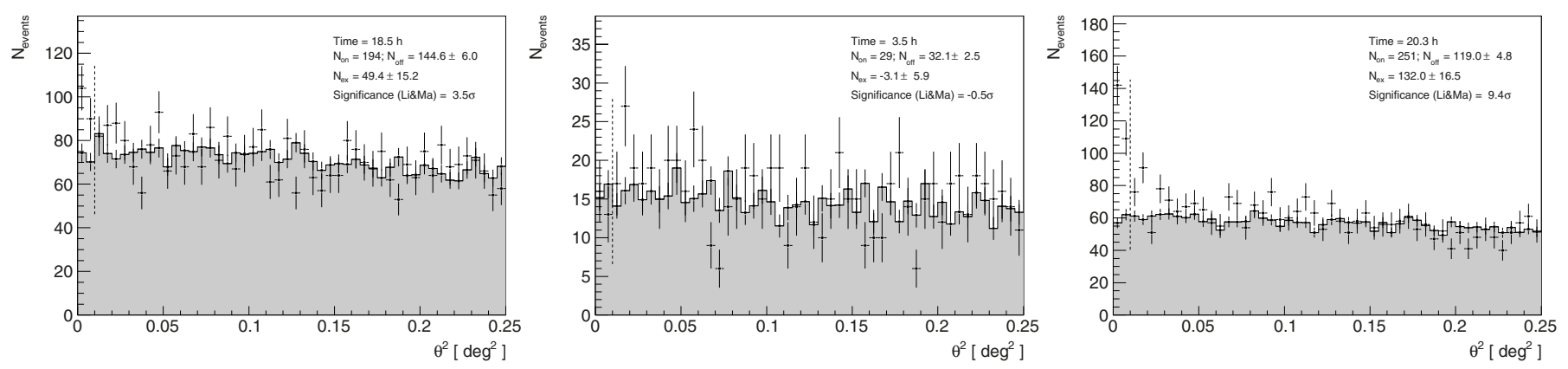

Fig. 1. Distributions of the $\theta^{2}$ parameter for 1ES 1215+303 signal (black histograms) and background estimation (gray histograms) for the three observation periods: January-February 2010 (left), May-June 2010 (middle), and January-February 2011 (right). The vertical dashed line corresponds to the apriori defined signal region $\theta^{2}<0.01 \mathrm{deg}^{2}$.

\section{Results}

\subsection{MAGIC results}

The MAGIC data were divided in three samples corresponding to three observation epochs: 2010 January-February, 2010 May-June, and 2011 January-February. The so-called $\theta^{2}$ plots (the distribution of the squared angular distance between the arrival direction of the events and the real position), for energies above $300 \mathrm{GeV}$, corresponding to the three observation epochs, are shown in Fig. 1. The computation of the number of the ON (signal) and OFF (background) events was performed in a fiducial signal region of $\theta^{2}<0.01 \mathrm{deg}^{2}$, and using 5 background regions (4 in case of the January-February 2010 data). In 2010 January-February (left panel) 194 ON events were detected over $144.6 \pm 6.0$ OFF events, with a significance level of $3.5 \sigma$ (using Eq. (17) in Li \& Ma 1983). In 2010 May-June (middle panel) the observation time was much shorter and no excess events were present. For 2011 data (right panel) the numbers were $251 \mathrm{ON}$ over $119 \pm 4.8$ OFF corresponding to a $\sim 9.4 \sigma$ significance, which is the first detection of VHE $\gamma$-rays from this source.

In Fig. 2, we show the significance map of the sky region for energies above $300 \mathrm{GeV}$ for the 2010 (January-February and May-June combined) and 2011 observations. 1ES 1218+304 is clearly visible in both maps while 1ES $1215+303$ was fainter in 2010 than in 2011 . The 1 ES $1218+304$ data analysis and results will be addressed in a separate paper.

For the light curve and spectrum determinations softer cuts were applied that have a higher $\gamma$-ray efficiency. The light curve (in a 5-days bins) above $200 \mathrm{GeV}$ of the 2011 data is well described by a constant flux of $(7.7 \pm 0.9) \times 10^{-12} \mathrm{~cm}^{-2} \mathrm{~s}^{-1}$ $\left(\chi^{2} / n_{\text {d.o.f. }}=0.56 / 3\right)$, which corresponds to about $3.5 \%$ of the Crab Nebula flux. Assuming that the hint of a signal seen in the 2010 data is a $\gamma$-ray excess the corresponding flux was $F(>200 \mathrm{GeV})=(3.4 \pm 1.0) \times 10^{-12} \mathrm{~cm}^{-2} \mathrm{~s}^{-1}$, which is less than half of the flux measured in 2011. The hypothesis of constant flux between 2010 and 2011 is excluded at the level of $3.1 \sigma$.

The derived VHE $\gamma$-ray spectrum for the 2011 observations can be described by a single power law $\left(\chi^{2} / n_{\text {d.o.f. }}=5.2 / 3\right.$, see Fig. 3):

$\frac{\mathrm{d} N}{\mathrm{~d} E}=(2.27 \pm 0.25) \times 10^{-11}\left(\frac{E}{300 \mathrm{GeV}}\right)^{(-2.96 \pm 0.14)} \mathrm{TeV}^{-1} \mathrm{~cm}^{-2} \mathrm{~s}^{-1}$

in the fitting range $70 \mathrm{GeV}-1.8 \mathrm{TeV}$. Since the spectral index of $1 E S 1215+303$ is similar to that of the Crab Nebula and the source is relatively bright, we can directly use the systematic errors estimated in Aleksić et al. (2012c). The systematic error of
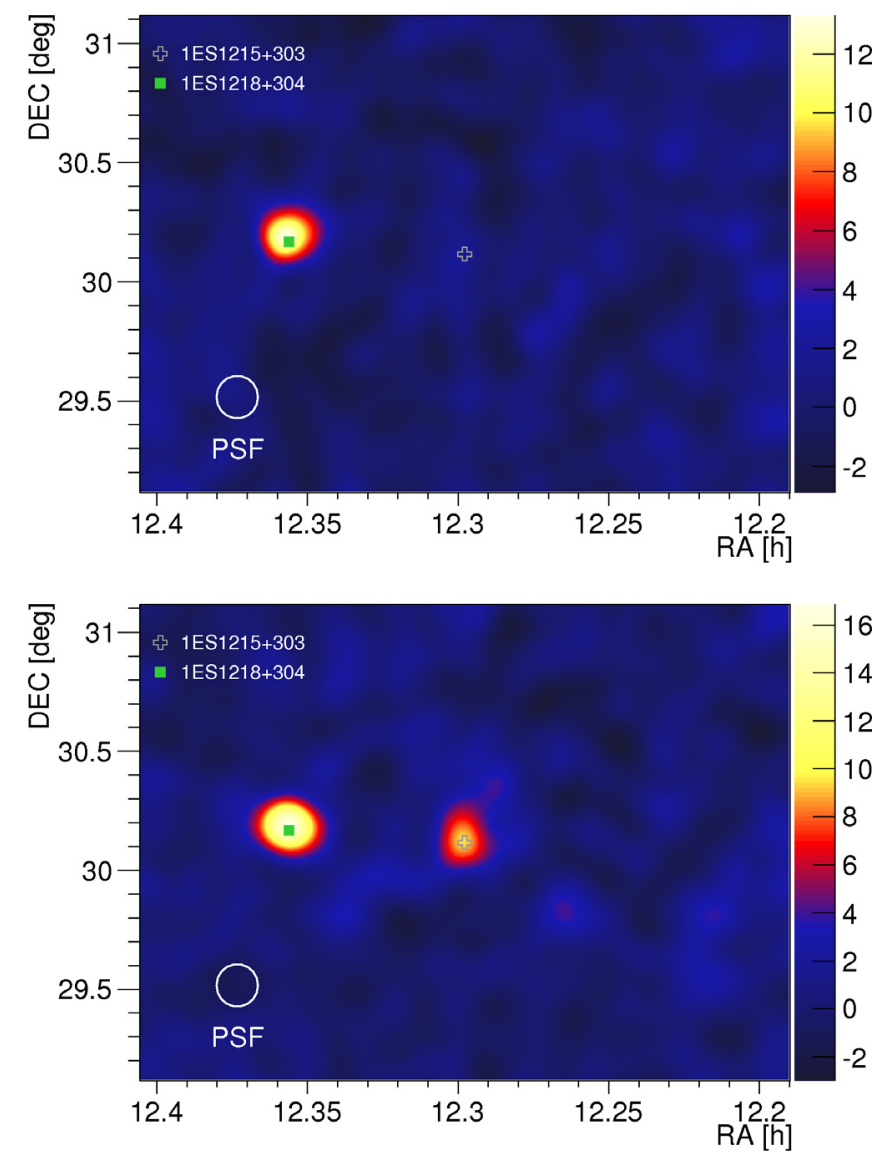

Fig. 2. Significance maps $(>300 \mathrm{GeV})$ from MAGIC observations performed during 2010 January-February and 2010 May-June (combined together, total time $22.0 \mathrm{~h}$, top) and 2011 January-February (total time $20.3 \mathrm{~h}$, bottom).

the slope is \pm 0.15 and in the energy range of the 1ES $1215+303$ spectrum, the error in the flux normalization (without the energy scale uncertainty) was estimated to be $11 \%$. The systematic error in the energy scale is $15 \%$. Finally, the MAGIC spectrum was deabsorbed using different EBL models (Domínguez et al. 2011; Kneiske \& Dole 2010; Franceschini et al. 2008; Primack et al. 2005) and the maximum high UV EBL model described in Albert et al. (2008) for $z=0.130$. The results are shown in Fig. 3. As denoted in the figure by the shaded area, at this redshift the EBL models agree well. The EBL model of Domínguez et al. (2011) was used to calculate the final intrinsic spectrum since this model is based on an observational approach. 
Table 1. Data summary and results for the Swift/XRT ToO observations.

\begin{tabular}{lccccc}
\hline \hline MJD & Obs. time $[\mathrm{ks}]$ & Flux $(2-10 \mathrm{keV})\left[10^{-12} \mathrm{erg} / \mathrm{cm}^{2} / \mathrm{s}\right]$ & $a(\Gamma$ for PL $)$ & $b$ & $\chi_{\text {red }}^{2} / n_{\text {d.o.f. }}$ \\
\hline 55168.6799 & 4.99 & $1.21 \pm 0.19$ & $2.56 \pm 0.10$ & $0.34 \pm 0.34$ & $1.19 / 25$ \\
55565.0340 & 4.39 & $2.74 \pm 0.25$ & $2.41 \pm 0.08$ & $0.37 \pm 0.24$ & $0.87 / 42$ \\
55569.1281 & 2.38 & $3.02 \pm 0.40$ & $2.29 \pm 0.16$ & - & $1.23 / 18(\mathrm{PL})$ \\
55571.1327 & 4.07 & $1.69 \pm 0.17$ & $2.65 \pm 0.14$ & - & $1.23 / 18(\mathrm{PL})$ \\
55572.1361 & 4.27 & $1.45 \pm 0.20$ & $2.64 \pm 0.09$ & $0.28 \pm 0.27$ & $1.15 / 32$ \\
55573.1396 & 2.99 & $1.73 \pm 0.25$ & $2.46 \pm 0.11$ & $0.66 \pm 0.37$ & $1.26 / 25$ \\
55674.2438 & 2.34 & $1.30 \pm 0.30$ & $2.67 \pm 0.25$ & - & $0.48 / 8(\mathrm{PL})$ \\
\hline
\end{tabular}

Notes. The datasets in the first/last rows are prior/subsequent to the MAGIC observations and are reported for comparison. For each dataset the following quantities are reported: the MJD time of the beginning of the observations; the exposure time; the integral flux in the 2-10 keV band; the $a$ and $b$ parameters for the log parabola fit (or the photon index $\Gamma$ in case a simple power-law is used, see text); the reduced $\chi^{2}$ with number of degrees of freedom $n_{\text {d.o.f. }}$. PL indicates when the simple power law is used instead of the log parabola.

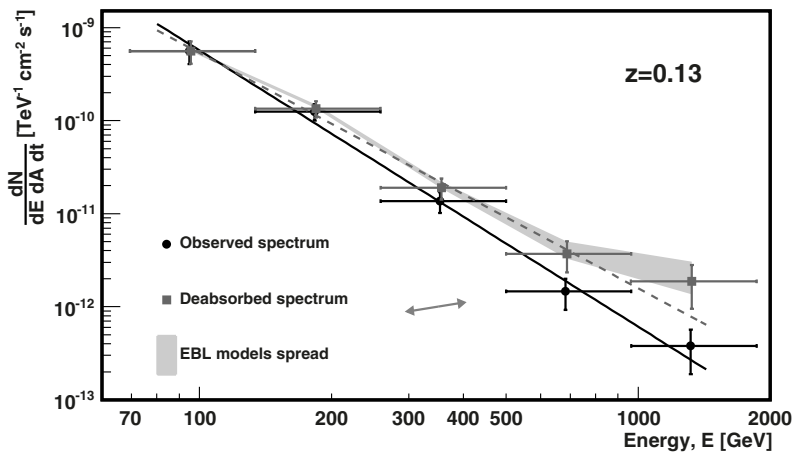

Fig. 3. Observed and deabsorped VHE $\gamma$-ray spectra. The EBL model of Domínguez et al. (2011) was used, the gray area shows the spread of the EBL models. The arrow shows the systematic error of the measurement.

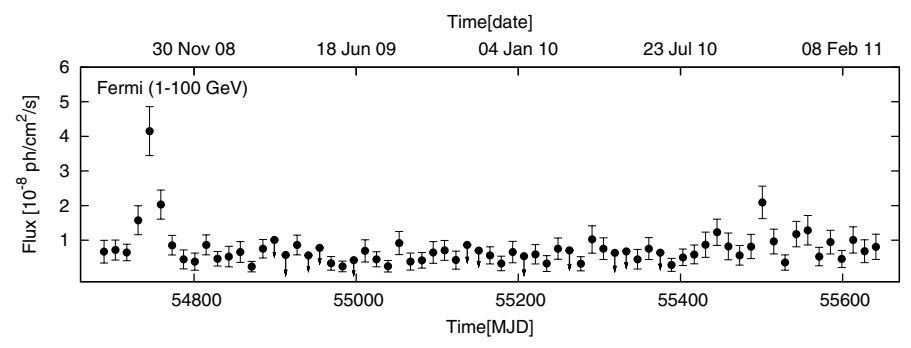

Fig. 4. Long-term light curve of 1ES 1215+303 from Fermi-LAT between 1 and $100 \mathrm{GeV}$.

\subsection{Fermi-LAT results}

The light curve of 1ES $1215+303$ was obtained in the energy range from 1 to $100 \mathrm{GeV}$, in 14-day bins from 2008 August to 2011 March (Fig. 4). It shows the major flare reported in Abdo et al. (2009) at the beginning of the Fermi mission. There is a hint of enhanced flux during 2010 November (MJD 55500 , duration only one bin, i.e. 14 days) but very little variability otherwise, especially at the two MAGIC observation epochs (2010 January-June and 2011 January-February). To maximize the number of photons the SED was derived using the whole MAGIC dataset (2010 January-June and 2011 January-February). The SEDs are shown in Fig. 5. In 2010 January-June the integral flux, $F(1-100 \mathrm{GeV})$ is $(4.9 \pm$ $0.7) \times 10^{-9} \mathrm{~cm}^{-2} \mathrm{~s}^{-1}$ and the photon index $2.1 \pm 0.1$, while in 2011 January-February $F(1-100 \mathrm{GeV})=(7.3 \pm 1.6) \times$ $10^{-9} \mathrm{~cm}^{-2} \mathrm{~s}^{-1}$ and the photon index $2.0 \pm 0.2$. The mean detected flux was $\sim 50 \%$ higher in 2011 January-February than in 2010 January-June, but due to large error bars the increase was not
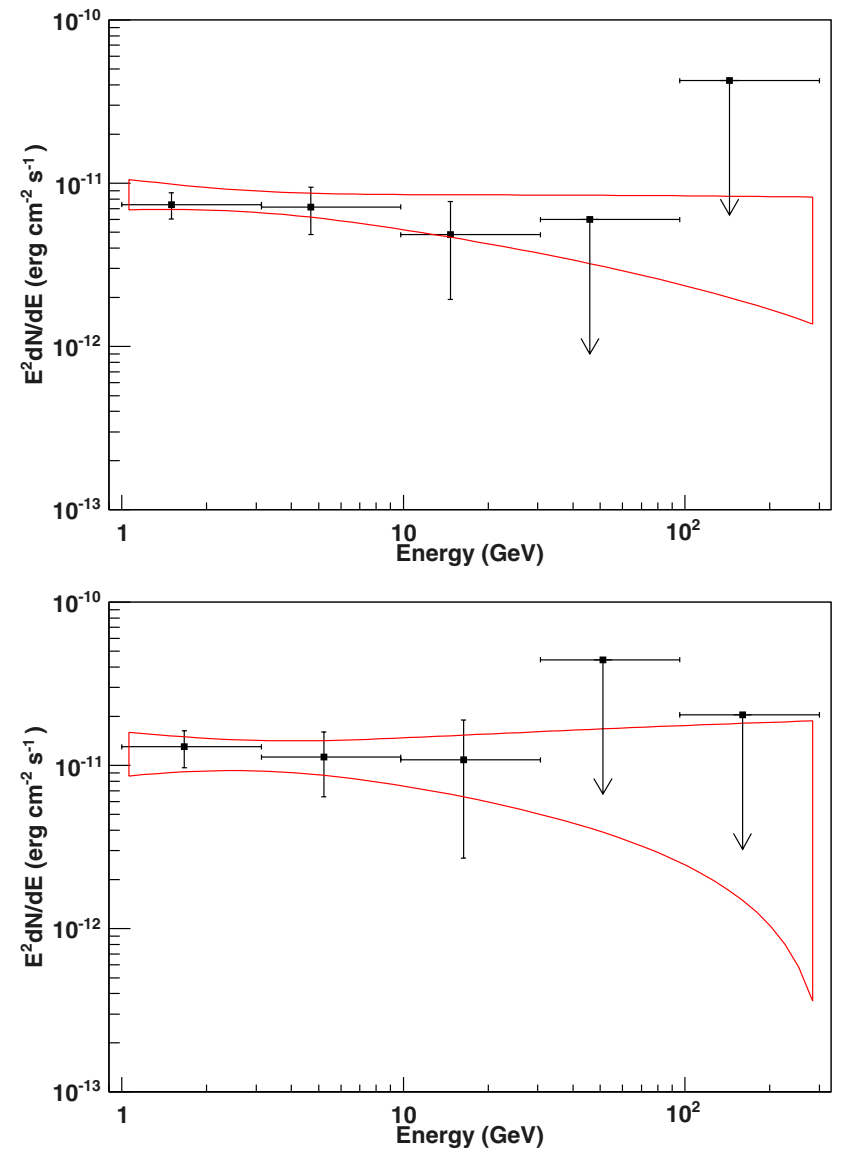

Fig. 5. Spectral energy distribution from Fermi-LAT derived for 2011 January-June (top) and 2011 January-February (bottom). The upper limits have been computed when the test statistics (see e.g. Mattox et al. 1996) in the energy band were lower than 4 . The bow-ties are derived from the unbinned likelihood analysis.

statistically significant. The spectral index was constant within the error bars.

\subsection{Swift results}

The results of the Swift/XRT observations are summarized in Table 1. The source showed the highest flux on 2011 January 8 (MJD 55 569.1) and previous/subsequent observations from 2009 December (MJD 55 168.7)/ 2011 April (MJD 55 674.2) show significantly lower flux. For the X-ray spectra both $\log$ parabola (in the form $\sim E^{-a-b * \log (E)}$, with $E$ being the energy in $\mathrm{keV}$ ) and a simple power-law fit were tested. The best fit was 
achieved with a log parabola law model in the range $0.3-10 \mathrm{keV}$ for four observations while a simple power law, in the range 0.5-10 keV, provided a better fit for three of the observations. Generally, a log parabolic fit suggests that there is curvature in the X-ray spectra but for 1ES $1215+303$, the difference between log parabolic and power law fits is small so no strong conclusions can be drawn. Because of the different fits a comparison between the spectral slopes is difficult, but for the highest flux night the spectral index is marginally harder than for the low state observations.

The Swift/UVOT results from 2011 January ToO observations show constant brightness with $V$-band magnitude $=$ $15.06 \pm 0.10, B=15.38 \pm 0.10, U=14.53 \pm 0.08, U V W 1=$ $14.43 \pm 0.08, U V M 2=14.35 \pm 0.06$, and $U V W 2=14.46 \pm 0.06$. However, in all bands the source is clearly brighter than in the previous observation (2009 December: $V=15.60 \pm 0.10$, $B=15.95 \pm 0.10, U=15.12 \pm 0.08, U V W 1=15.07 \pm 0.08$, $U V M 2=15.00 \pm 0.06$, and $U V W 2=15.15 \pm 0.06)$.

\subsection{KVA and Metsähovi results}

In the optical $R$-band the source is clearly variable on daily and yearly time-scales. The host galaxy contributes a flux of $0.99 \pm 0.09 \mathrm{mJy}$ (Nilsson et al. 2007) and when this contribution was subtracted from the measured flux, the AGN core was found to be $\sim 40 \%$ brighter in 2011 January-February (average total flux $3.64 \mathrm{mJy}$ ) than in 2010 January-June (average total flux $2.55 \mathrm{mJy}$ ). Similarly, it was found that during the 2011 January-February observations the flux varied by $\sim 25 \%$ (core flux between $3.2 \mathrm{mJy}$ and $4.1 \mathrm{mJy}$ ).

During 2011 January the optical polarization was $~ 9 \%$ while during the follow up observation in 2011 April it was higher, $\sim 15 \%$. The position angle (PA) was only slightly variable between 140 and 150 degrees.

In the radio band the source is rather weak and does not show strong variability. The $37 \mathrm{GHz}$ flux from the Metsähovi radio telescope has a similar level (0.3-0.4 Jy) in 2010 and 2011, although there were no radio observations during 2011 January-February.

\section{Interpretation}

In this section we discuss the quasi-simultaneous light curves, showing how they establish connections between different energy regimes and locate the emission region. The SED is reconstructed for the first time from radio frequencies to $\mathrm{TeV}$ energies for 1ES $1215+303$, allowing us to study the capability of the one-zone synchrotron self Compton model to reproduce the constructed SED.

\subsection{Multi-wavelength behavior}

The long-term multi-wavelength light curve, from radio to VHE $\gamma$-rays, is shown in Fig. 6. The MAGIC light curve shows a lower flux in 2010 (January-February and May-June) than in 2011 (by a factor of 2). The large uncertainties in the FermiLAT measurement do not allow us to conclusively say whether a similar flux enhancement also occurred in the $1-100 \mathrm{GeV}$ energy range (see Sect. 3.2). In X-rays the source was in a high state (enhanced by a factor of 2) in 2011 January compared with previous observations. In the optical band the average flux during MAGIC observations in 2010 was $3.5 \mathrm{mJy}$, while in 2011 it was $4.6 \mathrm{mJy}$. Thus, the source was clearly in outburst during early 2011 , at least in VHE $\gamma$-rays, X-rays, and the optical band.

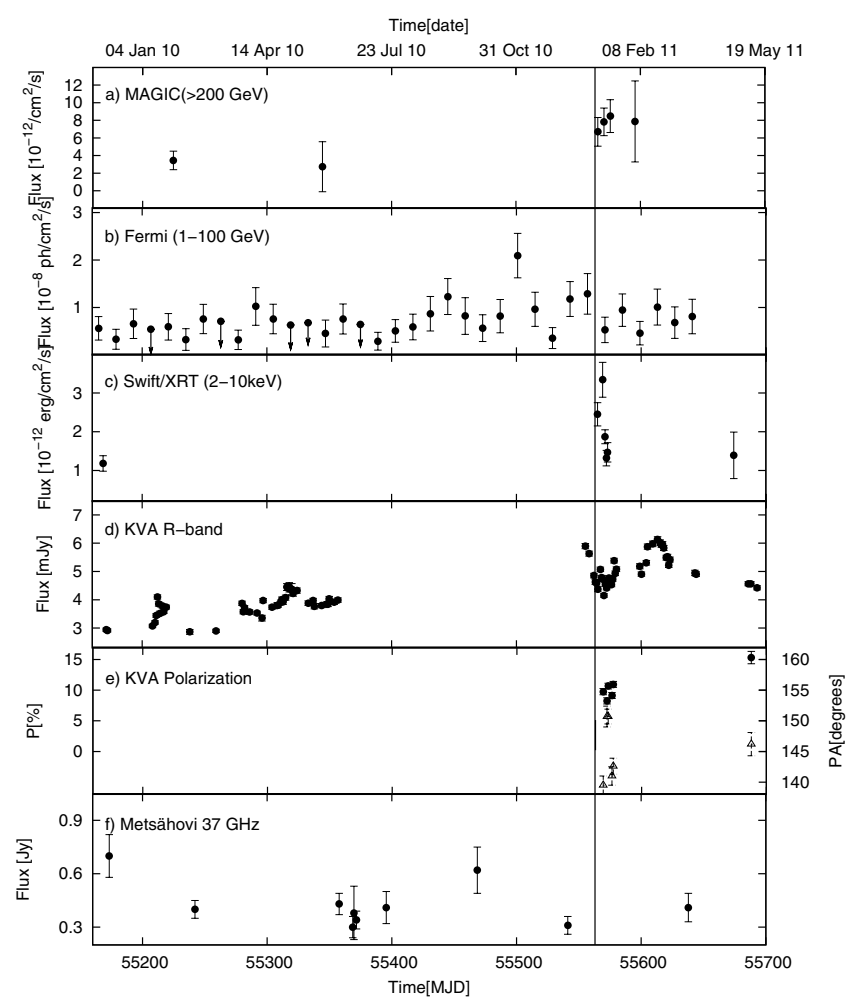

Fig. 6. Long-term multi-wavelength light curve of 1ES $1215+303$ from 2009 December to 2011 May. The vertical line shows the beginning of the MAGIC 2011 observation campaign. a) In the MAGIC light curve 2011 data are binned in 5-day intervals. 2010 data are divided in January-February and May-June bins. b) The Fermi-LAT light curve $(1-100 \mathrm{GeV})$ has bins of 14 days and the points with arrows are upper limits. c) The Swift/XRT light curve is derived from the target of opportunity observations performed during the MAGIC observations and archival data. d) The $R$-band light curve shows hourly average flux of the source, the error bars are smaller than the symbols in most cases. e) The optical polarization (filled circles, left axis) and polarization position angle (triangles, right axis) are hourly averages. f) $37 \mathrm{GHz}$ radio light curve from the Metsähovi radio observatory.

There were no simultaneous radio observations, but both the previous and subsequent observations showed low flux, suggesting that the outburst might have originated rather close to the central engine where the emission region is opaque at radio wavelengths. However, as the simultaneous observations are missing the existence of a simultaneous radio flare cannot be excluded.

During the 2011 January-February observations (Fig. 7), the MAGIC light curve is consistent with a constant flux. The source was in a rather low state in the Fermi-LAT energy range and no short term variability was detected. In X-rays and optical the source was variable during the MAGIC observations: the first two X-ray exposures gave a higher flux than for the latter three. The X-ray spectra show hints of hardening with higher flux, but they are statistically the same. The MAGIC observations started when the optical flux was decreasing, but during 2011 January the optical light curve showed several small flares. The X-ray light curve was more sparse and showed only one flare, but the comparison of simultaneous optical and X-ray points shows the same pattern in the light curves, indicating that the X-ray and optical emissions originates from the same region.

In addition to multi-wavelength variability studies, the optical polarization measurements have proven to be a powerful tool to analyze the emission scenarios in the blazar jets (e.g. Marscher et al. 2008). Polarization traces the magnetic field 


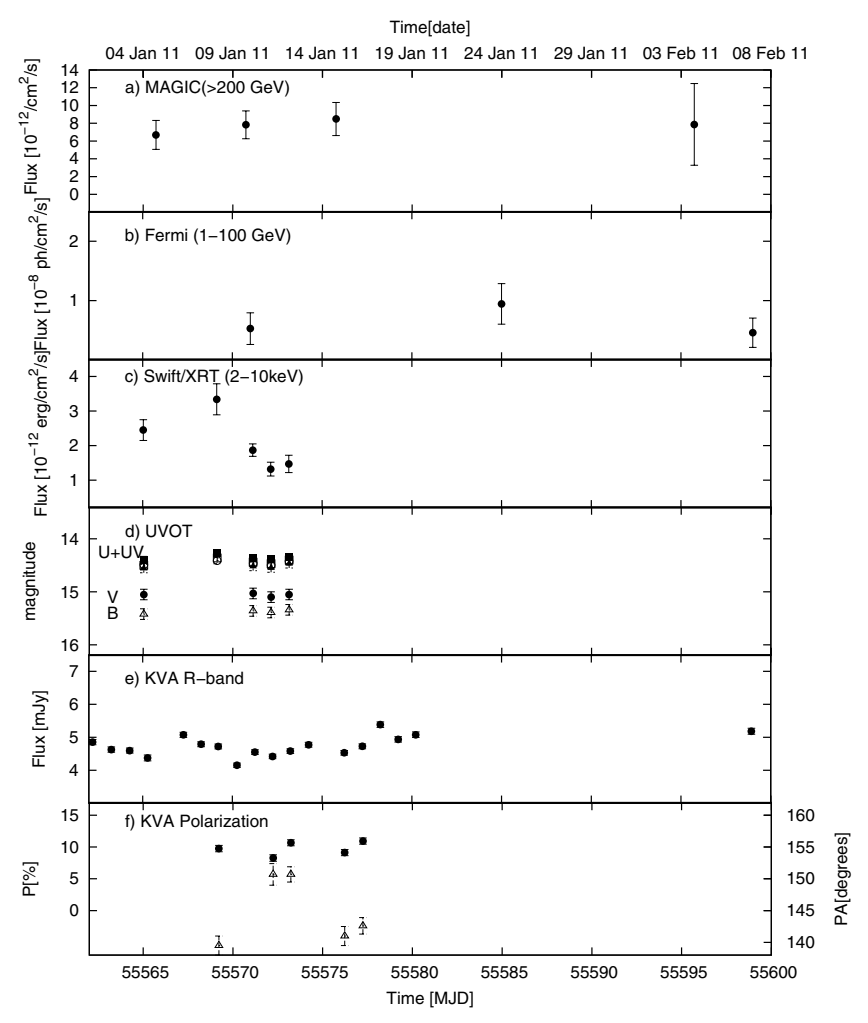

Fig. 7. Multi-wavelength light curve of 1ES 1215+303 from 2011 January to February. a) In the MAGIC light curve, the data are binned in 5-day intervals. b) The Fermi-LAT light curve $(1-100 \mathrm{GeV})$ has bins of 14 days. c) Swift/XRT light curve. d) UVOT optical and UV light curves. e) The $R$-band light curve shows hourly average fluxes of the source, the error bars are smaller than the symbols in most cases. f) The optical polarization (filled circles, left axis) and polarization position angle (triangles, right axis) are hourly averages.

of the jet. A net polarization oriented either parallel or perpendicular to the projected jet axis can be confused by shocks and the signatures are visible in optical polarization. The optical polarization measurements from 2011 January show little variability in polarization degree (average $\sim 9 \%$ ) or PA (varying between $\sim 140^{\circ}-150^{\circ}$ ) during the MAGIC observations, but the follow-up observations from 2011 April (Fig. 6) show a higher polarization, $\sim 15 \%$. Unfortunately, the polarization observations missed the peak of the first optical outburst and our data sample is very small. Ikejiri et al. (2011) monitored the photo-polarimetric behavior of the source in 2008-2009 and their observations seem to show similar polarization trends (i.e. a decreasing polarization during outbursts). They also found that the PA was almost constant at $\sim 150^{\circ}$, which agrees with our observations and with the historical data from 1981-1989 (Wills et al. 2011) showing PA values from $\sim 130-170^{\circ}$. Such preferred position angles have been observed for several BL Lac objects (e.g. Jannuzi et al. 1994) and implies long-term stability of the structure of the region producing the polarized emission e.g. the existence of a optical polarization core. In first order, if the optical outburst was produced by a shock traveling along the jet, one would expect the polarization degree to increase during the outburst. However, if there is a standing shock (optical polarization core) present, another shock with a different magnetic field orientation colliding with the standing component could produce an outburst in the total flux, but decrease the observed level of polarization (Villforth et al. 2010). A detailed photo-polarimetric study based on more data would be needed to further test this hypothesis.

\subsection{Spectral energy distribution}

The SED of 1ES $1215+303$ in both MAGIC observation epochs is shown in Fig. 8. The 2011 high energy bump is constructed using the MAGIC deabsorbed spectrum (using the EBL model of Domínguez et al. 2011) and the simultaneous Fermi-LAT spectrum (collecting all photons from 2011 January-February). As stated in Sect. 4.1, the low energy bump was variable during the period and is constructed for the night MJD 55569 that showed the highest Swift flux and for which there are simultaneous KVA and UVOT observations. The contribution of the host galaxy was subtracted from the $R$-band flux following Nilsson et al. (2007). The host galaxy also contaminates the $V, B$, and $U$ bands of the UVOT data, but its contribution should be negligible in the UV. As we have no direct measurements of the host galaxy contribution in $V, B$ and $U$ bands we extrapolated the magnitudes from the $R$-band value using the galaxy colors of elliptical galaxies at $z=0.2$ (Fukugita et al. 1995).

For the 2010 MAGIC data set, we could not derive a spectrum because of the low significance of the signal but we report the flux between $300 \mathrm{GeV}$ and $1 \mathrm{TeV}$ (assuming the same spectral index as in 2011). The simultaneous Fermi-LAT spectrum was calculated for the whole interval from 2010 January to June. There was no simultaneous X-ray observation, while for the optical we use the average (host galaxy subtracted) flux from nights when MAGIC was also observing. This "low state SED" is presented for illustrative purposes only but was not modeled, since both the synchrotron and IC peaks are poorly constrained.

The SED of 2011 shows two peaks, with the synchrotron peak frequency slightly above the optical band, as found for many other VHE $\gamma$-ray emitting BL Lac objects. The X-ray spectral index is also typical for a BL Lac source. The second peak seems to be located between the Fermi-LAT and MAGIC points $(\sim 1 \mathrm{GeV})$ as for many of the VHE $\gamma$-ray emitting BL Lacs. The locations of the synchrotron and IC peaks agree with values derived in Abdo et al. (2010a) for this source, but the synchrotron peak luminosity was slightly higher than in the previous observation by Giommi et al. (2012).

The emission characteristics of BL Lac objects is generally well reproduced by the one-zone leptonic model, in which a population of relativistic electrons inside a region moving down the jet emit through synchrotron and synchrotron self-Compton mechanisms (Bloom \& Marscher 1996; Tavecchio et al. 1998). The SED in 2011 is modeled with the one-zone leptonic model fully described in Maraschi \& Tavecchio (2003). The emission region was assumed to be spherical, with radius $R$, filled with a tangled magnetic field of intensity $B$ and relativistic electrons, emitting synchrotron and synchrotron self-Compton radiation. The electrons follow a smoothed broken power law energy distribution with normalization $K$ between $\gamma_{\min }$ and $\gamma_{\max }$, with slopes $n_{1}$ and $n_{2}$ below and above the break at $\gamma_{\mathrm{b}}$. The relativistic boosting is fully accounted for by the Doppler factor $\delta$. We note, however, that one-zone models cannot reproduce the spectrum at the lowest frequencies, since the emission is selfabsorbed below the millimeter band. It is generally assumed that this part of the SED is due to outer regions of the jet that is not important for the modeling of the high-energy emission.

The optical-UV and X-ray data define a narrow synchrotron component peaking around $10^{15} \mathrm{~Hz}$. At high energies, the SSC bump is well constrained by the Fermi-LAT and MAGIC data to peak at about $10 \mathrm{GeV}$. This particular structure of the SED is not easy to reproduce. In particular, the relatively wide separation between the two peaks inevitably implies a large value of the Doppler factor if standard parameters are 
Table 2. Input model parameters for the three models shown in Fig. 8.

\begin{tabular}{lcccccccccc}
\hline \hline Model & $\begin{array}{c}\gamma_{\min } \\
{\left[10^{3}\right]}\end{array}$ & $\begin{array}{c}\gamma_{\mathrm{b}} \\
{\left[10^{4}\right]}\end{array}$ & $\begin{array}{c}\gamma_{\max } \\
{\left[10^{6}\right]}\end{array}$ & $n_{1}$ & $n_{2}$ & $\begin{array}{c}B \\
{[\mathrm{G}]}\end{array}$ & $\begin{array}{c}K \\
{\left[\mathrm{~cm}^{-3}\right]}\end{array}$ & $\begin{array}{c}R \\
{\left[10^{16} \mathrm{~cm}\right]}\end{array}$ & $\delta$ & $\chi^{2} /$ d.o.f. \\
\hline high $\delta$ (dashed) & 1 & 3 & 1.0 & 2.0 & 4.2 & 0.02 & $8 \times 10^{3}$ & 0.8 & 60 & 3.36 \\
high $\gamma_{\min }$ (solid) & 8 & 9.2 & 2.5 & 3.0 & 4.85 & 0.055 & $1.3 \times 10^{8}$ & 1.0 & 30 & 6.94 \\
min $\chi^{2}$ (long dashed) & 1 & 1.6 & 16.1 & 1.8 & 3.7 & 0.01 & $3.22 \times 10^{2}$ & 3.75 & 36 & 1.04 \\
\hline
\end{tabular}

Notes. The following quantities are reported: the minimum, break, and maximum Lorentz factors and the low and high energy slope of the electron energy distribution, the magnetic field intensity, the electron density, the radius of the emitting region and its Doppler factor. In addition in the last column we report the $\chi^{2}$ d.o.f. assuming $2 \%, 10 \%$ and $40 \%$ systematical errors for optical-X-ray, GeV $\gamma$-rays and VHE $\gamma$-rays respectively.

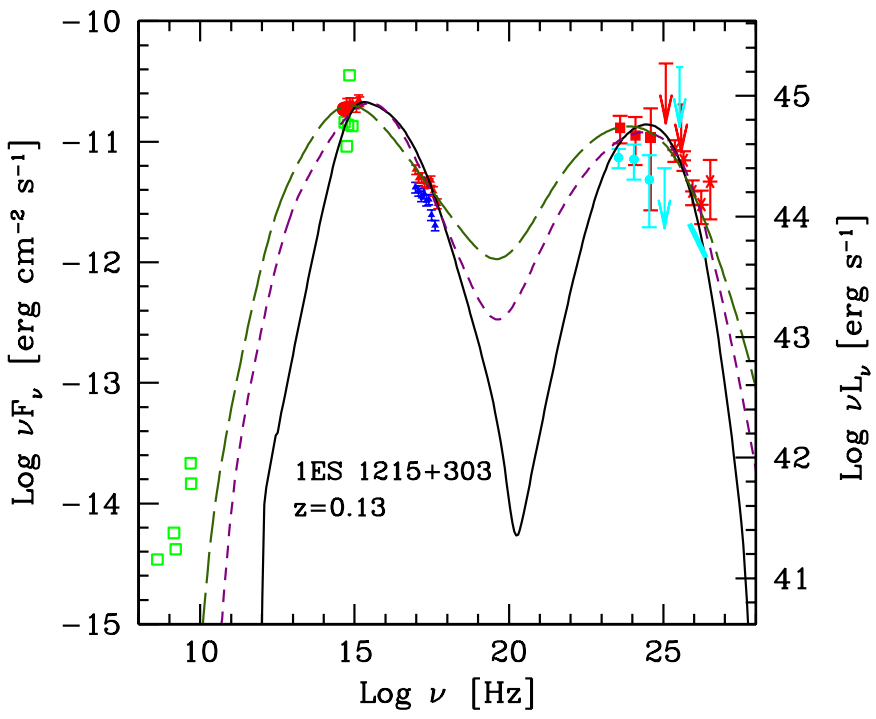

Fig. 8. Spectral energy distribution of 2011 January-February data (dark (red) symbols) modeled with the one-zone SSC model of Maraschi \& Tavecchio (2003). From high to low energies: the deabsorbed MAGIC spectra (asterisk, see text), the Fermi-LAT data (filled squares), Swift/XRT and Swift/UVOT data (triangles: upper (red) for MJD 55 569, lower (blue) for MJD 55 565) and simultaneous KVA data (filled circle, host galaxy subtracted, see text). The light (cyan) symbols report the January-June data of Fermi-LAT (data points and arrows) and MAGIC (the thick oblique line). The (green) open squares are archival data. The dashed line is the model fit using the extreme Doppler factor $\delta=60$, while the solid line is the model fit with high $\gamma_{\min }$ and the long dashed line reports the model parameters that produces with smallest $\chi^{2}$ (see text and Table 2).

used for the electron energy distribution (e.g. Georganopoulos \& Kazanas 2003; Tavecchio \& Ghisellini 2008). Our best attempt to reproduce the data in the standard framework provides the parameters given in Table 2 and is displayed as the dashed line of Fig. 8. As expected from the discussion above, we find a large Doppler factor, $\delta=60$, well above the typical range of Doppler factors obtained from the modeling of the emission of similar sources (e.g. Tavecchio et al. 2010) and disagreeing with the lower values required by the FR I-BL Lac $^{6}$ unification scheme (Urry \& Padovani 1995). However, there is an alternate, viable way to reproduce the observed SED using smaller Doppler factors; assume a relatively large minimum Lorentz factor of the emitting electrons, $\gamma_{\min }=8 \times 10^{3}$. This, along with a steep high energy electron energy distribution $\left(n_{2}=4.85\right)$, allows us to properly reproduce the narrow synchrotron bump and to locate the SSC peak at high enough energies using a moderately large boosting, $\delta=30$. This solution resembles one discussed

\footnotetext{
${ }^{6}$ Fanaroff-Riley I radio galaxies (FR I).
}

for the case of BL Lacs showing hard spectra in the soft X-ray and TeV band (Katarzyński et al. 2005; Tavecchio et al. 2009; Kaufmann et al. 2011; Lefa et al. 2011). Interestingly, such parameters (large $\gamma_{\mathrm{min}}$, steep slope) are consistent with the prediction of some simulations of particle acceleration by relativistic shocks (e.g. Virtanen \& Vainio 2003; Sironi \& Spitkovsky 2011). For example, for a proton-electron composition, it is expected that the electrons are heated when crossing the shock to a typical Lorentz factor of $\Gamma=\Gamma_{\text {rel }} m_{\mathrm{p}} / m_{\mathrm{e}}$, where $m_{\mathrm{p}} / m_{\mathrm{e}}=1836$ is the proton to electron mass ratio and $\Gamma_{\text {rel }}=2-3$ is the relative Lorentz factor between the upstream and the downstream flows. From this $\Gamma$ (that is equivalent to our parameter $\gamma_{\min }$ ), electrons are subsequently accelerated, forming a non-thermal tail that is well approximated by a steep $(n=3.5)$ power law.

The goodness of the fit can be judged by eye or by $\chi^{2}$-minimization procedure. For the fits presented above the "eye estimate" was used, as for the latter the systematic errors of the data from different instruments are in the key role. However, we also tested the automatic $\chi^{2}$-minimization procedure of Mankuzhiyil et al. (2011) with estimated systematical errors of $2 \%, 10 \%$ and $40 \%$ for optical-X-ray, $\mathrm{GeV} \gamma$-rays and VHE $\gamma$-rays respectively. The $\gamma_{\min }$ is fixed to same value as in our high $\delta$ model $\left(10^{3}\right)$ to allow easier comparison. The resulting parameters are shown in Table 2 and the fit with long-dashed (dark green) line in Fig. 8. The minimal $\chi^{2}$ fit results in lower $\delta$, but in a high $\gamma_{\max }$ and rather large emission region radius $R$ compared to other fits, but still compatible with the day scale variability observed in X-rays and optical.

\section{Summary and conclusions}

In this paper the first detection of VHE $\gamma$-rays from 1ES $1215+303$, resulting from MAGIC observations triggered by an optical outburst of the source in 2011 January, has been reported. In those data, the source is clearly detected at a $9.4 \sigma$ significance level. Also simultaneous multi-wavelength data are presented from radio to $\mathrm{HE} \gamma$-rays and compared to results from earlier MAGIC observations in 2010, when the source was in a lower optical state. The VHE $\gamma$-ray flux in 2011 was higher compared to 2010, suggesting that the activity in these two bands is connected. This conclusion is further supported by the fact that 1 ES $1215+303$ is already the fifth discovery at VHE $\gamma$-rays achieved after the MAGIC observations were triggered by an optical outburst.

Our collected multi-wavelength data set is the most extensive energy coverage for 1ES $1215+303$ to date. The opticalVHE $\gamma$-ray outburst seems to have been accompanied by an X-ray outburst, while in the Fermi-LAT band the flux increased only marginally. The optical photo-polarimetric data suggests that the high state could be caused by a shock traveling down the jet that collides with a standing shock with a differently oriented 
magnetic field. The X-ray and VHE $\gamma$-ray high states could then also originate from this collision.

The SED of 1ES $1215+303$ in 2011 January was model using a one-zone SSC model since it provides a good description of the SED of many VHE $\gamma$-ray emitting BL Lac objects. However, for 1ES $1215+303$ the synchrotron and IC peaks are narrow, the separation between the two peaks is wide, and a simple one-zone SSC model with typical parameters failed to reproduce the observed SED. To fit the SED, a high Doppler factor or a narrow electron energy distribution is required. While high Doppler factors are disfavored by the unified models, the high $\gamma_{\min }$ value could be a viable solution in the light of simulations modeling the acceleration of electrons in a relativistic shock in a protonelectron jet. This should be further investigated, e.g. using the fully self-consistent SSC model with particle acceleration due to shock and stochastic acceleration by Weidinger et al. (2010); Weidinger \& Spanier (2010).

Given the rather extreme conditions needed for the onezone model, the presence of a velocity structure in the jet (Georganopoulos \& Kazanas 2003; Ghisellini et al. 2005) is also possible and should be tested for modeling the SED. The narrow synchrotron and IC peaks should well constrain the model. Additionally, the more complex emission scenario suggested by the photo-polarimetric behavior of 1ES $1215+303$ should be tested using, for instance, a model of Marscher (2011), who investigated the emission from a turbulent ambient jet plasma that passes through either the standing or moving shocks in the jet. Further observations of this exceptional VHE $\gamma$-ray emitting BL Lac are strongly encouraged.

Acknowledgements. We would like to thank the Instituto de Astrofísica de Canarias for the excellent working conditions at the Observatorio del Roque de los Muchachos in La Palma. The support of the German BMBF and MPG, the Italian INFN, the Swiss National Fund SNF, and the Spanish MICINN is gratefully acknowledged. This work was also supported by the Marie Curie program, by the CPAN CSD2007-00042 and MultiDark CSD200900064 projects of the Spanish Consolider-Ingenio 2010 programme, by grant DO02-353 of the Bulgarian NSF, by grant 127740 of the Academy of Finland, by the YIP of the Helmholtz Gemeinschaft, by the DFG Cluster of Excellence "Origin and Structure of the Universe", by the DFG Collaborative Research Centers SFB823/C4 and SFB876/C3, and by the Polish MNiSzW grant 745/NHESS-MAGIC/2010/0. The Metsähovi team acknowledges the support from the Academy of Finland to our observing projects (numbers 212656, 210338, 121148, and others). The Fermi-LAT Collaboration acknowledges generous ongoing support from a number of agencies and institutes that have supported both the development and the operation of the LAT as well as scientific data analysis. These include the National Aeronautics and Space Administration and the Department of Energy in the United States, the Commissariat à l'Énergie Atomique and the Centre National de la Recherche Scientifique/Institut National de Physique Nucléaire et de Physique des Particules in France, the Agenzia Spaziale Italiana and the Istituto Nazionale di Fisica Nucleare in Italy, the Ministry of Education, Culture, Sports, Science and Technology (MEXT), High Energy Accelerator Research Organization (KEK) and Japan Aerospace Exploration Agency (JAXA) in Japan, and the K. A. Wallenberg Foundation, the Swedish Research Council and the Swedish National Space Board in Sweden. Additional support for science analysis during the operations phase is gratefully acknowledged from the Instituto Nazionale di Astrofisica in Italy and the Centre National d'Études Spatiales in France.

\section{References}

Abdo, A. A., Ackermann, M., Ajello, M., et al. 2009, ApJ, 700, 597 Abdo, A. A., Ackermann, M., Agudo, I., et al. 2010a, ApJ, 716, 30 Abdo, A. A., Ackermann, M., Ajello, M., et al. 2010b, ApJ, 722, 520 Acciari, V. A., Aliu, E., Arlen, T., et al. 2011, ApJ, 738, 25 Ackermann, M., Ajello, M., Allafort, A., et al. 2011, ApJ, 743, 171 Akiyama, M., Ueda, Y., Ohta, K., Takahashi, T., \& Yamada, T. 2003, ApJS, 148, 275

Albert, J., Aliu, E., Anderhub, H., et al. 2006a, ApJ, 642, L119 Albert, J., Aliu, E., Anderhub, H., et al. 2006b, ApJ, 648, L105 Albert, J., Aliu, E., Anderhub, H., et al. 2007, ApJ, 667, L21
Albert, J., Aliu, E., Anderhub, H., et al. 2008, Science, 320, 1752

Aleksić, J., Antonelli, L. A., Antoranz, P., et al. 2011a, ApJ, 729, 115

Aleksić, J., Antonelli, L. A., Antoranz, P., et al. 2011b, A\&A, 530, A4

Aleksić, J., Alvarez, E. A., Antonelli, L. A., et al. 2012a, A\&A, 539, A118

Aleksić, J., Alvarez, E. A., Antonelli, L. A., et al. 2012b, ApJ, 748, 46

Aleksić, J., Alvarez, E. A., Antonelli, L. A., et al. 2012c, Astropart. Phys., 35, 435

Anderhub, H., Antonelli, L. A., Antoranz, P., et al. 2009, ApJ, 704, L129

Barthelmy, S. D., Barbier, L. M., Cummings, J. R., et al. 2005, Space Sci. Rev., 120,143

Bessell, M. S. 1979, PASP, 91, 589

Bloom, S. D., \& Marscher, A. P. 1996, ApJ, 461, 657

Burrows, D. N., Hill, J. E., Nousek, J. A., et al. 2005, Space Sci. Rev., 120, 165 Costamante, L., \& Ghisellini, G. 2002, A\&A, 384, 56

Dermer, C. D., \& Schlickeiser, R. 1993, ApJ, 416, 458

Domínguez, A., Primack, J. R., Rosario, D. J., et al. 2011, MNRAS, 410, 2556

Fiorucci, M., \& Tosti, G. 1996, A\&AS, 116, 403

Fomin, V. P., Fennell, S., Lamb, R. C., et al. 1994, Astropart. Phys., 2, 151

Franceschini, A., Rodighiero, G., \& Vaccari, M. 2008, A\&A, 487, 837

Fukugita, M., Shimasaku, K., \& Ichikawa, T. 1995, PASP, 107, 945

Gehrels, N., Chincarini, G., Giommi, P., et al. 2004, ApJ, 611, 1005

Georganopoulos, M., \& Kazanas, D. 2003, ApJ, 594, L27

Ghisellini, G., Tavecchio, F., \& Chiaberge, M. 2005, A\&A, 432, 401

Giommi, P., Polenta, G., Lahteenmaki, A., et al. 2012, A\&A, 541, A160

Horan, D., Badran, H. M., Bond, I. H., et al. 2004, ApJ, 603, 51

Ikejiri, Y., Uemura, M., Sasada, M., et al. 2011, PASJ, 63, 639

Jannuzi, B. T., Smith, P. S., \& Elston, R. 1994, ApJ, 428, 130

Kalberla, P. M. W., Burton, W. B., Hartmann, D., et al. 2005, A\&A, 440, 775

Katarzyński, K., Ghisellini, G., Tavecchio, F., et al. 2005, A\&A, 433, 479

Kaufmann, S., Wagner, S. J., Tibolla, O., \& Hauser, M. 2011, A\&A, 534, A130

Kneiske, T. M., \& Dole, H. 2010, A\&A, 515, A19

Lefa, E., Rieger, F. M., \& Aharonian, F. 2011, ApJ, 740, 64

Li, T.-P., \& Ma, Y.-Q. 1983, ApJ, 272, 317

Mankuzhiyil, N., Ansoldi, S., Persic, M., \& Tavecchio, F. 2011, ApJ, 733, 14

Maraschi, L., \& Tavecchio, F. 2003, ApJ, 593, 667

Maraschi, L., Ghisellini, G., \& Celotti, A. 1992, ApJ, 397, L5

Mariotti, M. 2011, The Astronomer's Telegram, 3100, 1

Marscher, A. P. 2011, in AAS Meeting Abstracts, 218, 327.07

Marscher, A. P., Jorstad, S. G., D’Arcangelo, F. D., et al. 2008, Nature, 452, 966

Mattox, J. R., Bertsch, D. L., Chiang, J., et al. 1996, ApJ, 461, 396

Nilsson, K., Pasanen, M., Takalo, L. O., et al. 2007, A\&A, 475, 199

Nolan, P. L., Abdo, A. A., Ackermann, M., et al. 2012, ApJS, 199, 31

Pei, Y. C. 1992, ApJ, 395, 130

Piirola, V., Berdyugin, A., Mikkola, S., \& Coyne, G. V. 2005, ApJ, 632, 576

Poole, T. S., Breeveld, A. A., Page, M. J., et al. 2008, MNRAS, 383, 627

Primack, J. R., Bullock, J. S., \& Somerville, R. S. 2005, in High Energy Gamma-Ray Astronomy, ed. F. A. Aharonian, H. J. Völk, \& D. Horns, AIP Conf. Ser., 745, 23

Rees, M. J. 1967, MNRAS, 137, 429

Reinthal, R., Lindfors, E. J., Mazin, D., et al. 2012, J. Phys. Conf. Ser., 355, 012013

Roming, P. W. A., Kennedy, T. E., Mason, K. O., et al. 2005, Space Sci. Rev., 120,95

Schlegel, D. J., Finkbeiner, D. P., \& Davis, M. 1998, ApJ, 500, 525

Sironi, L., \& Spitkovsky, A. 2011, ApJ, 726, 75

Tavecchio, F., \& Ghisellini, G. 2008, MNRAS, 385, L98

Tavecchio, F., Maraschi, L., \& Ghisellini, G. 1998, ApJ, 509, 608

Tavecchio, F., Ghisellini, G., Ghirlanda, G., Costamante, L., \& Franceschini, A. 2009, MNRAS, 399, L59

Tavecchio, F., Ghisellini, G., Ghirlanda, G., Foschini, L., \& Maraschi, L. 2010, MNRAS, 401, 1570

Teräsranta, H., Tornikoski, M., Mujunen, A., et al. 1998, A\&AS, 132, 305

Urry, C. M., \& Padovani, P. 1995, PASP, 107, 803

Villforth, C., Nilsson, K., Heidt, J., et al. 2010, MNRAS, 402, 2087

Virtanen, J., \& Vainio, R. 2003, in High Energy Blazar Astronomy, July 2002,

Piikkio, Finland, eds. L. O. Takalo, \& E. Valtaoja, ASP Conf. Proc., 299, 157

Weidinger, M., \& Spanier, F. 2010, A\&A, 515, A18

Weidinger, M., Rüger, M., \& Spanier, F. 2010, Astrophys. Space Sci. Trans., 6, 1

Wills, B. J., Wills, D., \& Breger, M. 2011, ApJS, 194, 19

\footnotetext{
1 IFAE, Edifici Cn., Campus UAB, 08193 Bellaterra, Spain

2 Universidad Complutense, 28040 Madrid, Spain

3 INAF National Institute for Astrophysics, 00136 Rome, Italy

4 Università di Siena, and INFN Pisa, 53100 Siena, Italy

5 Technische Universität Dortmund, 44221 Dortmund, Germany

6 Max-Planck-Institut für Physik, 80805 München, Germany
} 
7 Università di Padova and INFN, 35131 Padova, Italy

8 Inst. de Astrofísica de Canarias, 38200 La Laguna, Tenerife, Spain

9 University of Łódź, 90236 Lodz, Poland

10 Depto. de Astrofísica, Universidad de La Laguna, 38206 La Laguna, Spain

11 Deutsches Elektronen-Synchrotron (DESY), 15738 Zeuthen, Germany

12 ETH Zurich, 8093 Zurich, Switzerland

13 Universität Würzburg, 97074 Würzburg, Germany

14 Universitat de Barcelona (ICC/IEEC), 08028 Barcelona, Spain

15 Università di Udine, and INFN Trieste, 33100 Udine, Italy

16 Institut de Ciències de l'Espai (IEEC-CSIC), 08193 Bellaterra, Spain

17 Inst. de Astrofísica de Andalucía (CSIC), 18080 Granada, Spain

18 Croatian MAGIC Consortium, Rudjer Boskovic Institute, University of Rijeka and University of Split, 10000 Zagreb, Croatia

19 Universitat Autònoma de Barcelona, 08193 Bellaterra, Spain

20 Tuorla Observatory, University of Turku, 21500 Piikkiö, Finland

21 Inst. for Nucl. Research and Nucl. Energy, 1784 Sofia, Bulgaria

22 Japanese MAGIC Consortium, Division of Physics and Astronomy, Kyoto University, Japan

23 INAF/Osservatorio Astronomico and INFN, 34143 Trieste, Italy

24 Università dell'Insubria, Como, 22100 Como, Italy
25 Università di Pisa, and INFN Pisa, 56126 Pisa, Italy

${ }^{26}$ ICREA, 08010 Barcelona, Spain

27 Now at: École polytechnique fédérale de Lausanne (EPFL), Lausanne, Switzerland

28 Supported by INFN Padova

29 Now at: Centro de Investigaciones Energéticas, Medioambientales y Tecnológicas (CIEMAT), Madrid, Spain

30 Now at: KIPAC, SLAC National Accelerator Laboratory, USA

31 Now at: Institut für Experimentalphysik, University of Hamburg, Germany

32 Finnish Centre for Astronomy with ESO (FINCA), University of Turku, Finland

33 Aalto University Metsähovi Radio Observatory, Metsähovintie 114, 02540, Kylmälä, Finland

${ }^{34}$ Department of Physics, Stockholm University, AlbaNova, 10691 Stockholm, Sweden

35 The Oskar Klein Centre for Cosmoparticle Physics, AlbaNova, 106 91 Stockholm, Sweden

36 Department of Astronomy, Stockholm University, 10691 Stockholm, Sweden

*Corresponding authors: jbecerra@iac.es, elilin@utu.fi, colin@mppmu.mpg.de, saverio.lombardi@pd.infn.it, jsitarek@ifae.es 